PROCEEDINGS OF THE AMERICAN MATHEMATICAL SOCIETY

Volume 124, Number 8, August 1996

\title{
GENERALIZED CONTRACTION MAPPING PRINCIPLE AND DIFFERENTIAL EQUATIONS IN PROBABILISTIC METRIC SPACES
}

\author{
S. S. CHANG, B. S. LEE, Y. J. CHO, Y. Q. CHEN, S. M. KANG, AND J. S. JUNG \\ (Communicated by Palle E. T. Jorgensen)

\begin{abstract}
A new generalized contraction mapping principle in probabilistic metric spaces is obtained. As an application, we utilize this principle to prove the existence theorems of solutions to differential equations in probabilistic metric spaces. All the results presented in this paper are new.
\end{abstract}

\section{INTRODUCTION AND PRELIMINARIES}

The purpose of this paper is to obtain a new generalized contraction mapping principle in probabilistic metric spaces. As an application, we utilize this principle to study the existence problem of solutions for some kind of differential equations in probabilistic metric spaces. The results presented in this paper are all new.

For the sake of convenience, we first recall some definitions and notation in [3]$[5]$.

Throughout this paper, let $R=(-\infty,+\infty)$ and $R^{+}=[0,+\infty)$.

Definition 1. A mapping $F: R \rightarrow R^{+}$is called a distribution function if it is nondecreasing and left-continuous with $\inf _{t \in R} F(t)=0$ and $\sup _{t \in R} F(t)=1$.

In what follows we always denote by $\mathcal{D}$ the st of all distribution functions and by $H$ the specific distribution function defined by

$$
H(t)= \begin{cases}0, & \text { if } t \leq 0 \\ 1, & \text { if } t>0 .\end{cases}
$$

Definition 2. A probabilistic metric space (briefly, a PM-space) is an ordered pair $(E, \mathcal{F})$, where $E$ is a nonempty set and $\mathcal{F}$ is a mapping from $E \times E$ into $\mathcal{D}$. We denote the distribution function $\mathcal{F}(x, y)$ by $F_{x, y}$, and $F_{x, y}(t)$ represents the value of $F_{x, y}$ at $t \in R$. The function $F_{x, y}$ is assumed to satisfy the following conditions: for all $x, y \in E$,

(PM-1) $F_{x, y}(t)=1$ for all $t>0$ if and only if $x=y$,

$(\mathrm{PM}-2) F_{x, y}(0)=0$,

(PM-3) $F_{x, y}(t)=F_{y, x}(t)$ for all $t \in R$,

(PM-4) if $F_{x, y}\left(t_{1}\right)=1$ and $F_{y, z}\left(t_{2}\right)=1$, then $F_{x, z}\left(t_{1}+t_{2}\right)=1$.

Received by the editors January 3, 1995

1991 Mathematics Subject Classification. Primary 46S50, 34G20, 54H25.

Key words and phrases. Generalized contraction mapping, probabilistic metric space, probabilistic normed space, probabilistic bounded set.

(C)1996 American Mathematical Society 
Definition 3. A mapping $\Delta:[0,1] \times[0,1] \rightarrow[0,1]$ is called a $t$-norm if it satisfies the following conditions: for any $a, b, c, d \in[0,1]$,

(T-1) $\Delta(a, 1)=a$,

(T-2) $\Delta(a, b)=\Delta(b, a)$,

(T-3) $\Delta(c, d) \geq \Delta(a, b)$ for $c \geq a$ and $d \geq b$,

(T-4) $\Delta(\Delta(a, b), c)=,\Delta(a, \Delta(b, c))$.

Definition 4. A Menger PM-space is a triplet $(E, \mathcal{F}, \Delta)$, where $(E, \mathcal{F})$ is a PMspace and $\Delta$ is a $t$-norm satisfying the following triangle inequality: for all $x, y, z \in$ $E$ and $t_{1}, t_{2} \geq 0$,

$$
F_{x, z}\left(t_{1}+t_{2}\right) \geq \Delta\left(F_{x, y}\left(t_{1}\right), F_{y, z}\left(t_{2}\right)\right) .
$$

Schweizer, Sklar and Thorp [5] proved that if $(E, \mathcal{F}, \Delta)$ is a Menger PM-space with $\sup _{0<t<1} \Delta(t, t)=1$, then $(E, \mathcal{F}, \Delta)$ is a Hausdorff topological space in the topology $\mathcal{T}$ induced by the family of $(\varepsilon, \lambda)$-neighborhoods

$$
\left\{U_{p}(\varepsilon, \lambda): p \in E, \varepsilon>0, \lambda>0\right\},
$$

where

$$
U_{p}(\varepsilon, \lambda)=\left\{x \in E: F_{x, p}(\varepsilon)>1-\lambda\right\} .
$$

Definition 5. A triplet $(E, \mathcal{F}, \Delta)$ is called a Menger probabilistic normed space (briefly, a Menger PN-space) if $E$ is a real vector space, $\mathcal{F}$ is a mapping from $E$ into $\mathcal{D}$ (for $x \in E$, the distribution function $\mathcal{F}(x)$ is denoted by $F_{x}$ and $F_{x}(t)$ is the value of $F_{x}$ at $t \in R$ ) and $\Delta$ is a $t$-norm satisfying the following conditions:

$(\mathrm{PN}-1) F_{x}(0)=0$,

(PN-2) $F_{x}(t)=H(t)$ for all $t>0$ if and only if $x=0$,

(PN-3) $F_{\alpha x}(t)=F_{x}\left(\frac{t}{|\alpha|}\right)$ for all $\alpha \in R, \alpha \neq 0$,

$(\mathrm{PN}-4) F_{x+y}\left(t_{1}+t_{2}\right) \geq \Delta\left(F_{x}\left(t_{1}\right), F_{y}\left(t_{2}\right)\right)$ for all $x, y \in E$ and $t_{1}, t_{2} \in R^{+}$.

Definition 6. Let $(E, \mathcal{F}, \Delta)$ be a Menger PM-space with $\sup _{0<t<1} \Delta(t, t)=1$.

(1) A sequence $\left\{x_{n}\right\}$ in $E$ is said to be $\mathcal{T}$-convergent to $x \in E$ (we write $x_{n} \stackrel{\mathcal{T}}{\rightarrow} x$ ) if for any given $\varepsilon>0$ and $\lambda>0$, there exists a positive integer $N=N(\varepsilon, \lambda)$ such that $F_{x_{n}, x}(\varepsilon)>1-\lambda$ whenever $n \geq N$.

(2) A sequence $\left\{x_{n}\right\}$ in $E$ is called a $\mathcal{T}$-Cauchy sequence if for any $\varepsilon>0$ and $\lambda>0$, there exists a positive integer $N=N(\varepsilon, \lambda)$ such that $F_{x_{n}, x_{m}}(\varepsilon)>1-\lambda$, whenever $n, m \geq N$.

(3) A Menger PM-space $(E, \mathcal{F}, \Delta)$ is said to be $\mathcal{T}$-complete if each $\mathcal{T}$-Cauchy sequence in $E$ is $\mathcal{T}$-convergent to some point in $E$.

\section{A generalized CONTRACTION MAPPING PRINCIPLE}

Theorem 1. Let $(E, \mathcal{F}, \Delta)$ be a complete Menger PM-space with a $t$-norm $\Delta$ satisfying $\Delta(t, t) \geq t$ for all $t \in[0,1]$. Let $T: E \rightarrow E$ be a mapping satisfying the following condition:

$$
F_{T x, T y}(t) \geq F_{x, y}(t / k(\alpha, \beta))
$$

for all $x, y \in E, t \geq 0$ and $\alpha, \beta \in(0,+\infty)$ with $F_{x, y}(\alpha)>0$ and $F_{x, y}(\beta)<1$, where $k(\alpha, \beta):(0,+\infty)^{2} \rightarrow(0,1)$ is a function.

Then $T$ has exactly one fixed point in $E$. 
In the sequel the mapping $T$ which satisfies (2.1) is called a generalized contraction mapping on $E$.

To prove the conclusion of Theorem 1, we need the following lemma:

Lemma $([2$, Theorem 1]). Let $(E, \mathcal{F}, \Delta)$ be a Menger PM-space, $\Delta$ be a $t$-norm satisfying $\lim _{t \rightarrow 1-} \Delta(t, s)=s$ for all $s \in[0,1]$ and $\left\{p_{n}\right\},\left\{q_{n}\right\}$ be two sequences in E with $p_{n} \stackrel{\mathcal{T}}{\rightarrow} p, q_{n} \stackrel{\mathcal{T}}{\rightarrow} q$, respectively.

(1) For any given $t \in R, \liminf _{n \rightarrow \infty} F_{p_{n}, q_{n}}(t) \geq F_{p, q}(t)$.

(2) If $t \in R$ is a continuous point of $F_{p, q}$, then $\lim _{n \rightarrow \infty} F_{p_{n}, q_{n}}(t)=F_{p, q}(t)$.

Proof of Theorem 1. (1) First we prove that for any given $\varepsilon>0$ and $\lambda \in(0,1)$ there exist $x_{0} \in E$ and an $(\varepsilon, \lambda)$-neighborhood of $x_{0}, N\left(x_{0}, \varepsilon, \lambda\right)=\left\{x \in E: F_{x, x_{0}}(\varepsilon)>\right.$ $1-\lambda\}$, such that $T: N\left(x_{0}, \varepsilon, \lambda\right) \rightarrow N\left(x_{0}, \varepsilon, \lambda\right)$.

Suppose the contrary. Then there exist $\varepsilon_{0}>0$ and $\lambda_{0} \in(0,1)$ such that for each $x \in E$, there is an $x_{1} \in E$ with

$$
F_{x, x_{1}}\left(\varepsilon_{0}\right)>1-\lambda_{0}, \quad F_{T x_{1}, x}\left(\varepsilon_{0}\right) \leq 1-\lambda_{0} .
$$

(a) If $F_{x, x_{1}}\left(\frac{\varepsilon_{0}}{2}\right)>1-\frac{\lambda_{0}}{2}$, then

$$
F_{x, T x_{1}}\left(\varepsilon_{0}\right) \geq \Delta\left(F_{x, T x}\left(\frac{\varepsilon_{0}}{2}\right), F_{T x, T x_{1}}\left(\frac{\varepsilon_{0}}{2}\right)\right) .
$$

If $x_{1}=x$, then $F_{T x, T x_{1}}\left(\frac{\varepsilon_{0}}{2}\right)=1$. By (2.2) and (2.3), we have

$$
F_{x, T x}\left(\frac{\varepsilon_{0}}{2}\right) \leq F_{x, T x_{1}}\left(\varepsilon_{0}\right) \leq 1-\lambda_{0} .
$$

If $x_{1} \neq x$, then there exists $t_{0}>0$ such that $F_{x, x_{1}}\left(t_{0}\right)<1$. By condition $(2.1)$ and the assumption of (a), we have

$$
F_{T x, T x_{1}}\left(\frac{\varepsilon_{0}}{2}\right) \geq F_{x, x_{1}}\left(\varepsilon_{0} / 2 k\left(\frac{\varepsilon_{0}}{2}, t_{0}\right)\right) \geq F_{x, x_{1}}\left(\frac{\varepsilon_{0}}{2}\right)>1-\frac{\lambda_{0}}{2} .
$$

From (2.2) and (2.3), we have

$$
\Delta\left(F_{x, T x}\left(\frac{\varepsilon_{0}}{2}\right), 1-\frac{\lambda_{0}}{2}\right) \leq \Delta\left(F_{x, T x}\left(\frac{\varepsilon_{0}}{2}\right), F_{T_{x}, T_{x_{1}}}\left(\frac{\varepsilon_{0}}{2}\right)\right) \leq F_{x, T_{x_{1}}}\left(\varepsilon_{0}\right) \leq 1-\lambda_{0}
$$

Therefore we have

$$
F_{x, T x}\left(\frac{\varepsilon_{0}}{2}\right) \leq 1-\lambda_{0}
$$

(b) If $F_{x, x_{1}}\left(\frac{\varepsilon_{0}}{2}\right) \leq 1-\frac{\lambda_{0}}{2}$, then by the condition (2.1), we have

$$
\begin{aligned}
F_{x, T x_{1}}\left(\varepsilon_{0}\right) & \geq \Delta\left[F_{x, T x}\left(\left(1-k\left(\frac{\varepsilon_{0}}{2}, \varepsilon_{0}\right)\right) \varepsilon_{0}\right), F_{T x, T x_{1}}\left(k\left(\frac{\varepsilon_{0}}{2}, \varepsilon_{0}\right) \varepsilon_{0}\right)\right] \\
& \geq \Delta\left(F_{x, T x}\left(\left(1-k\left(\frac{\varepsilon_{0}}{2}, \varepsilon_{0}\right)\right) \varepsilon_{0}\right), F_{x, x_{1}}\left(\varepsilon_{0}\right)\right) .
\end{aligned}
$$

By (2.2), we have

$$
1-\lambda_{0} \geq F_{x, T_{x_{1}}}\left(\varepsilon_{0}\right) \geq \Delta\left(F_{x, T_{x}}\left(\left(1-k\left(\frac{\varepsilon_{0}}{2}, \varepsilon_{0}\right)\right) \varepsilon_{0}\right), 1-\lambda_{0}\right)
$$

and so we have

$$
F_{x, T x}\left(\left(1-k\left(\frac{\varepsilon_{0}}{2}, \varepsilon_{0}\right)\right) \varepsilon_{0}\right) \leq 1-\lambda_{0}
$$


Letting

$$
\eta_{0}=\min \left\{\frac{\varepsilon_{0}}{2},\left(1-k\left(\frac{\varepsilon_{0}}{2}, \varepsilon_{0}\right)\right) \varepsilon_{0}\right\}
$$

by $(2.5)$ we have

$$
F_{x, T x}\left(\eta_{0}\right) \leq 1-\lambda_{0}, \quad x \in E .
$$

Besides, for any fixed $x_{0} \in E$, since $\lim _{t \rightarrow+\infty} F_{x_{0}, T x_{0}}(t)=1$, there exists a $t_{1}>0$ such that $F_{x_{0}, T x_{0}}\left(t_{1}\right)>0$. It is easy to see that

$$
F_{T^{n} x_{0}, T^{n+1} x_{0}}(t) \geq F_{x_{0}, T x_{0}}\left(t / k^{n}\left(\eta_{0}, t_{0}\right)\right), \quad t \geq 0, n=1,2, \ldots
$$

Taking $t=\eta_{0}$, we have

$$
F_{T^{n} x_{0}, T^{n+1} x_{0}}\left(\eta_{0}\right) \geq F_{x_{0}, T x_{0}}\left(\eta_{0} / k^{n}\left(\eta_{0}, t_{0}\right)\right), \quad n=1,2, \ldots
$$

Letting $n$ be large enough so that $F_{x_{0}, T x_{0}}\left(\eta_{0} / k^{n}\left(\eta_{0}, t_{0}\right)\right)>1-\lambda_{0}$, we have

$$
F_{T^{n} x_{0}, T^{n+1} x_{0}}\left(\eta_{0}\right)>1-\lambda_{0},
$$

which contradicts (2.6). The desired conclusion is proved.

Because $T$ satisfies condition (2.1), $T$ is continuous and so

$$
T: \overline{N\left(x_{n}, \varepsilon, \lambda\right)} \rightarrow \overline{N\left(x_{0}, \varepsilon, \lambda\right)} .
$$

(2) For given $\varepsilon_{n}>0$ and $\lambda_{n} \in(0,1), n=1,2, \ldots$, with $\varepsilon_{n}>\varepsilon_{n+1}, \lambda_{n}>$ $\lambda_{n+1}, \varepsilon_{n} \rightarrow 0^{+}$and $\lambda_{n} \rightarrow 0^{+}$, by the conclusion proved in (1), there exist $x_{n} \in E$ and a $\left(\varepsilon_{n}, \lambda_{n}\right)$-neighborhood $N\left(x_{n}, \varepsilon_{n}, \lambda_{n}\right)$ of $x_{n}$ such that

$$
T: \overline{N\left(x_{n}, \varepsilon_{n}, \lambda_{n}\right)} \rightarrow \overline{N\left(x_{n}, \varepsilon_{n}, \lambda_{n}\right)}, \quad n=1,2 \ldots .
$$

It is obvious that $\overline{N\left(x_{n}, \varepsilon_{n}, \lambda_{n}\right)} \supseteq \overline{N\left(x_{n+1}, \varepsilon_{n+1}, \lambda_{n+1}\right)}$ for all $n \geq 1$. Therefore, we have

$$
T: \bigcap_{n \geq 1} \overline{N\left(x_{n}, \varepsilon_{n}, \lambda_{n}\right)} \rightarrow \bigcap_{n \geq 1} \overline{N\left(x_{n}, \varepsilon_{n}, \lambda_{n}\right)} .
$$

Next, we prove that $\bigcap_{n>1} \overline{N\left(x_{n}, \varepsilon_{n}, \lambda_{n}\right)} \neq \varnothing$. In fact, for any given $\varepsilon>0, \lambda>0$ and $n$ large enough such that $\varepsilon_{n}<\varepsilon, \lambda_{n}<\lambda$, take $t_{n, m} \in\left(\varepsilon_{n}, \varepsilon\right)$ such that $t_{n, m}$ is a continuous point of $F_{x_{n}, x_{n+m}}(t)$. Since $x_{n+m} \in \overline{N\left(x_{n}, \varepsilon_{n}, \lambda_{n}\right)}$, by the lemma, we have

$$
F_{x_{n}, x_{n+m}}(\varepsilon) \geq F_{x_{n}, x_{n+m}}\left(t_{n, m}\right) \geq 1-\lambda_{n}>1-\lambda .
$$

This implies that $\left\{x_{n}\right\}$ is a $\mathcal{T}$-Cauchy sequence in $E$. Let $x_{n} \stackrel{\mathcal{T}}{\rightarrow} x$. Then we have

$$
x \in \bigcap_{n \geq 1} \overline{N\left(x_{n}, \varepsilon_{n}, \lambda_{n}\right)} .
$$

The desired conclusion is proved.

(3) Finally, we prove that $\bigcap_{n \geq 1} \overline{N\left(x_{n}, \varepsilon_{n}, \lambda_{n}\right)}$ has only one point.

Suppose the contrary. Then there exist $x, y \in \bigcap_{n \geq 1} \overline{N\left(x_{n}, \varepsilon_{n}, \lambda_{n}\right)}$ with $x \neq y$ and hence there exist $t_{0}>0$ and $\lambda_{0} \in(0,1)$ such that $F_{x, y}\left(t_{0}\right)<\lambda_{0}$. Letting $n$ be large enough so that $\varepsilon_{n}<t_{0} / 2$ and $1-\lambda_{n}>\lambda_{0}$, then we have

$$
F_{x, y}\left(t_{0}\right) \geq \Delta\left(F_{x, x_{n}}\left(\frac{t_{0}}{2}\right), F_{x_{n}, y}\left(\frac{t_{0}}{2}\right)\right) \text {. }
$$


Taking $t_{n} \in\left(\varepsilon_{n}, \frac{t_{0}}{2}\right)$ and $s_{n} \in\left(\varepsilon_{n}, \frac{t_{0}}{2}\right)$ such that $t_{n}$ is a continuous point of $F_{x, x_{n}}(t)$ and $s_{n}$ is a continuous point of $F_{x_{n}, y}(t)$, then we have

$$
F_{x, x_{n}}\left(t_{n}\right) \geq 1-\lambda_{n}, \quad F_{x_{n}, y}\left(s_{n}\right) \geq 1-\lambda_{n}
$$

and so $F_{x, y}\left(t_{0}\right)>\lambda_{0}$, which is a contradiction. Therefore, $\bigcap_{n>1} \overline{N\left(x_{n}, \varepsilon_{n}, \lambda_{n}\right)}$ has only one point, say $x_{*}$, and it is just the unique fixed point of $T$. This completes the proof.

In order to give the other result, we first give the following definition ([1]):

Definition 7. Let $(E, \mathcal{F}, \Delta)$ be a Menger PM-space and $A$ be a subset of $E$. $A$ is said to be probabilistically bounded if

$$
\sup _{t>0} \inf _{x, y \in A} F_{x, y}(t)=1 .
$$

Theorem 2. Let $(E, \mathcal{F}, \Delta)$ be a complete Menger $P N$-space, $C$ be a probabilistically bounded closed convex subset of $E, T: C \rightarrow E$ be a generalized contraction mapping defined on $C$ and $S: C \rightarrow E$ be a continuous mapping with $S(C)$ being relatively compact. If $T x+S y \in C$ for all $x, y \in C$, then $T+S$ has a fixed point in $C$.

Proof. For any given $y \in C$, we know that $T x+S y: C \rightarrow C$ is a generalized contraction mapping. By Theorem 1, $T x+S y$ has exactly one fixed point $x_{(y)} \in C$ such that

$$
x_{(y)}=T x_{(y)}+S y .
$$

Now we define a mapping $K$ by $K y=x_{(y)}$ for all $y \in C$, where $x_{(y)}$ is the unique fixed point of $T x+S y$. It is obvious that $K$ is a continuous mapping from $C$ into $C$ and $K(C)$ is relatively compact. Therefore there exists $y_{*} \in C$ such that $y_{*}=K y_{*}$. Taking $y=y_{*}$ in (2.8), we have

$$
Y_{*}=K y_{*}=x_{\left(y_{*}\right)}=T x_{\left(y_{*}\right)}+S y_{*},
$$

i.e., $T y_{*}+S y_{*}=y_{*}$. This completes the proof.

\section{Differential EQUations in PROBabilistic NORMED SPACES}

In this section we shall use the results in the section 2 to show the existence of solutions of a class of differential equations in Menger PN-spacs.

Let $(E, \mathcal{F}, \Delta)$ be a Menger PN-space and $C([0, T], E)$ be a set of mappings $x(\cdot):[0, T] \rightarrow E$ which are continuous in the topology $\mathcal{T}$ on $(E, \mathcal{F}, \Delta)$. Define a mapping $\widetilde{\mathcal{F}}: C([0, T], E) \rightarrow \mathcal{D}$ by

$$
\widetilde{\mathcal{F}}_{x(\cdot)}(t)=\lim _{k \rightarrow t-s \in[0, T]} \inf _{x(s)}(k)
$$

and denote the distribution function $\widetilde{\mathcal{F}}_{x(\cdot)}$ by $\widetilde{F}_{x(\cdot)}$ and the value of $\widetilde{\mathcal{F}}_{x(\cdot)}$ at $t \in R$ by $\widetilde{F}_{x(\cdot)}(t)$.

We have the following results about $(C([0, T], E), \widetilde{\mathcal{F}}, \Delta)$ :

Proposition 3. Let $(E, \mathcal{F}, \Delta)$ be a Menger PN-space with a continuous t-norm $\Delta$. Then $(C([0, T], E), \widetilde{\mathcal{F}}, \Delta)$ is also a Menger PN-space.

Proof. (I) First we prove that $\widetilde{\mathcal{F}}$ is a mapping from $C([0, T], E)$ into $\mathcal{D}$. In fact, for any given $x(\cdot) \in C([0, T], E)$ :

1. It is obvious that $\widetilde{F}_{x(\cdot)}(t)$ is nondecreasing with $\inf _{t \in R} \widetilde{F}_{x(\cdot)}(t)=0$. 
2. Next we prove that $\widetilde{F}_{x(\cdot)}(t)$ is left-continuous.

In fact, since $\inf _{s \in[0, T]} F_{x(s)}(t)$ is increasing in $t$, for any given $t_{n} \in R$ with $t_{n} \rightarrow t-$, we have

$$
\begin{aligned}
\varlimsup_{t_{n} \rightarrow t-} \widetilde{F}_{x(\cdot)}\left(t_{n}\right) & =\varlimsup_{t_{n} \rightarrow t-}\left(\lim _{k \rightarrow t_{n}-}\left(\inf _{s \in[0, T]} F_{x(s)}(k)\right)\right) \\
& \leq \varlimsup_{t_{n} \rightarrow t-}\left(\inf _{s \in[0, T]} F_{x(s)}\left(t_{n}\right)\right) \\
& =\lim _{t_{n}-t-}\left(\inf _{s \in[0, T]} F_{x(s)}\left(t_{n}\right)\right) \\
& =\widetilde{F}_{x(\cdot)}(t) .
\end{aligned}
$$

Letting $\left\{r_{n}\right\}$ be any sequence in $(0,+\infty)$ such that $r_{n} \rightarrow 0^{+}$and noting again that $\inf _{s \in[0, T]} F_{x(s)}(t)$ is increasing in $t$, we have

$$
\widetilde{F}_{x(\cdot)}\left(t_{n}\right)=\lim _{k \rightarrow t_{n}-}\left(\inf _{s \in[0, T]} F_{x(s)}(k)\right) \geq \inf _{s \in[0, T]} F_{x(s)}\left(t_{n}-r_{n}\right) .
$$

Therefore we have

$$
\begin{aligned}
\varliminf_{t_{n} \rightarrow t-} \widetilde{F}_{x(\cdot)}\left(t_{n}\right) & \geq \varliminf_{t_{n} \rightarrow t-}\left(\inf _{s \in[0, T]} F_{x(s)}\left(t_{n}-r_{n}\right)\right) \\
& =\varlimsup_{u_{n} \rightarrow t-}\left(\inf _{s \in[0, T]} F_{x(s)}\left(u_{n}\right)\right) \quad\left(u_{n}=t_{n}-r_{n}\right) \\
& =\varlimsup_{u_{n} \rightarrow t-}\left(\inf _{s \in[0, T]} F_{x(s)}\left(u_{n}\right)\right) \\
& =\widetilde{F}_{x(\cdot)}(t) .
\end{aligned}
$$

Thus combining (3.1) and (3.2), it follows that

$$
\lim _{t_{n} \rightarrow t-} \widetilde{F}_{x(\cdot)}\left(t_{n}\right)=\widetilde{F}_{x(\cdot)}(t) \text {. }
$$

This implies that $\widetilde{F}_{x(\cdot)}(t)$ is left-continuous in $t$.

3. Finally we prove that $\sup _{t \in R} \widetilde{F}_{x(\cdot)}(t)=1$.

From (1) of Lemma and the continuity of $x(\cdot)$, we now that for each $k \in R^{+}$, there exists $s_{k} \in[0, T]$ such that

$$
\inf _{s \in[0, T]} F_{x(s)}(k) \geq F_{x\left(s_{k}\right)}(k) .
$$

Therefore we have

$$
\begin{aligned}
\varlimsup_{t \rightarrow+\infty} \widetilde{F}_{x(\cdot)}(t) & =\varlimsup_{t \rightarrow+\infty} \lim _{k \rightarrow t-}\left(\inf _{s \in[0, T]} F_{x(s)}(k)\right) \\
& \geq \varlimsup_{t \rightarrow+\infty}\left(\underline{\lim }_{k \rightarrow t-} F_{x\left(s_{k}\right)}(k)\right) .
\end{aligned}
$$

Without loss of generality, we can assume that $s_{k} \rightarrow s_{t}^{\prime}$ as $k \rightarrow t-$ (otherwise, we can choose a subsequence of $\left.\left\{s_{k}\right\}\right)$. Hence we have

$$
{\underline{\varliminf_{k \rightarrow t-}}}_{k x\left(s_{k}\right)}(k) \geq \underline{\lim }_{k \rightarrow t-} F_{x\left(s_{k}\right)}(t / 2) \geq F_{x\left(s_{t}^{\prime}\right)}(t / 2) .
$$

It follows from (3.4) and (3.5) that

$$
\varlimsup_{t \rightarrow+\infty} \widetilde{F}_{x(\cdot)}(t) \geq \varlimsup_{t \rightarrow+\infty} F_{x\left(s_{t}^{\prime}\right)}(t / 2) .
$$


Without loss of generality, we assume that $s_{t}^{\prime} \rightarrow s_{0}$ as $t \rightarrow+\infty$. Therefore, for any $\varepsilon \in(0, t / 2)$, from the condition (PN-4) in Definition 5 , we have

$$
F_{x\left(s_{t}^{\prime}\right)}\left(\frac{t}{2}\right) \geq \Delta\left(F_{x\left(s_{t}^{\prime}\right)-x\left(s_{0}\right)}(\varepsilon), F_{x(0)}\left(\frac{t}{2}-\varepsilon\right)\right) .
$$

Since

$$
\lim _{t \rightarrow+\infty} F_{x\left(s_{t}^{\prime}\right)-x\left(s_{0}\right)}(\varepsilon)=1
$$

it follows that

$$
\varlimsup_{t \rightarrow+\infty} F_{x\left(s_{0}\right)}\left(\frac{t}{2}-\varepsilon\right)=1 .
$$

From (3.6)-(3.9), we have

$$
\varlimsup_{t \rightarrow+\infty} \widetilde{F}_{x(\cdot)}(t)=1, \quad \text { i.e., } \sup _{t \in R} \widetilde{F}_{x(\cdot)}(t)=1 .
$$

(II) Next we prove that $\widetilde{F}$ satisfies all the conditions (PN-1)-(PN-4) in Definition 5.

In fact, it follows that

(i) $\widetilde{F}_{x(\cdot)}(0)=0$ for all $x(\cdot) \in C([0, T], E)$ is obvious.

(ii) $\widetilde{F}_{x(\cdot)}(t)=H(t)$ for all $t \in R$ if and only if $x(s)=0$ for all $s \in[0, T]$.

(iii) For any $\alpha \in R, \alpha \neq 0$,

$$
\begin{aligned}
\widetilde{F}_{\alpha x(\cdot)}(t) & =\lim _{k \rightarrow t-}\left(\inf _{s \in[0, T]} F_{\alpha x(s)}(k)\right) \\
& =\lim _{k \rightarrow t-}\left(\inf _{s \in[0, T]} F_{x(s)}(k /|\alpha|)\right) \\
& =\widetilde{F}_{x(\cdot)}(t /|\alpha|) .
\end{aligned}
$$

(iv) For all $x(\cdot), y(\cdot) \in(C[0, T], E)$ and $t_{1}, t_{2} \in R$,

$$
\begin{aligned}
\widetilde{F}_{x(\cdot)+y(\cdot)}\left(t_{1}+t_{2}\right) & =\lim _{\substack{k_{1} \rightarrow t_{1}-\\
k_{2} \rightarrow t_{2}-}}\left(\inf _{s \in[0, T]} F_{x(s)+y(s)}\left(k_{1}+k_{2}\right)\right) \\
& \geq \lim _{\substack{k_{1} \rightarrow t_{1}-\\
k_{2} \rightarrow t_{2}-}}\left(\inf _{s \in[0, T]} \Delta\left(F_{x(s)}\left(k_{1}\right), F_{y(s)}\left(k_{2}\right)\right)\right), \quad k_{1}, k_{2} \geq 0 .
\end{aligned}
$$

Since $F_{x(s)}\left(k_{1}\right) \geq \inf _{s \in[0, T]} F_{x(s)}\left(k_{1}\right)$ and $F_{y(s)}\left(k_{2}\right) \geq \inf _{s \in[0, T]} F_{y(s)}\left(k_{2}\right)$, we have

$$
\widetilde{F}_{x(\cdot)+y(\cdot)}\left(t_{1}+t_{2}\right) \geq \lim _{\substack{k_{1} \rightarrow t_{1}-\\ k_{2} \rightarrow t_{2}-}} \Delta\left(\inf _{s \in[0, T]} F_{x(s)}\left(k_{1}\right), \inf _{s \in[0, T]} F_{y(s)}\left(k_{2}\right)\right) .
$$

In view of the continuity of $\Delta$ and (3.10), we have

$$
\widetilde{F}_{x(\cdot)+y(\cdot)}\left(t_{1}+t_{2}\right) \geq \Delta\left(\widetilde{F}_{x(\cdot)}\left(t_{1}\right), \widetilde{F}_{y(\cdot)}\left(t_{2}\right)\right) .
$$

This completes the proof.

Proposition 4. Let $(E, \mathcal{F}, \Delta)$ be a complete Menger PN-space with a continuous t-norm $\Delta$. Then $(C([0, T], E), \widetilde{\mathcal{F}}, \Delta)$ is also a complete Menger PN-space. 
Proof. In Proposition 3, we proved that $(C([0, T], E), \widetilde{\mathcal{F}}, \Delta)$ is a Menger PN-space. Now we prove that it is complete. Let $\left\{x_{n}(\cdot)\right\}$ be a Cauchy sequence in $(C([0, T], E), \widetilde{\mathcal{F}}, \Delta)$ such that

$$
x(t)=\lim _{n \rightarrow \infty} x_{n}(t), \quad t \in[0, T] .
$$

Now we prove that $x(\cdot) \in(C([0, T], E), \widetilde{\mathcal{F}}, \Delta)$. In fact, for any given $t_{0} \in[0, T]$ we have

$$
\begin{aligned}
\widetilde{F}_{x(t)-x\left(t_{0}\right)}(s) & \geq \Delta\left[\widetilde{F}_{x(t)-x_{n}(t)}\left(\frac{s}{2}\right), \widetilde{F}_{x_{n}(t)-x\left(t_{0}\right)}\left(\frac{s}{2}\right)\right] \\
& \geq \Delta\left[\widetilde{F}_{x(t)-x_{n}(t)}\left(\frac{s}{2}\right), \Delta\left[\widetilde{F}_{x_{n}(t)-x_{n}\left(t_{0}\right)}\left(\frac{\varepsilon}{4}\right), \widetilde{F}_{x_{n}\left(t_{0}\right)-x\left(t_{0}\right)}\left(\frac{\varepsilon}{4}\right)\right]\right] .
\end{aligned}
$$

By using $\left\{x_{n}(\cdot)\right\} \subset(C([0, T], E), \widetilde{\mathcal{F}}, \Delta)$ and $(3.11)$, we have

$$
x(\cdot) \in(C([0, T], E), \widetilde{\mathcal{F}}, \Delta) .
$$

Therefore $(C([0, T], E), \widetilde{\mathcal{F}}, \Delta)$ is complete. This completes the proof.

We are now in a position to show the existence of solutions of the following differential equation:

$$
\left\{\begin{array}{l}
x^{\prime}(t)=f(t, x)+g(t, x), \\
x(0)=x_{0}
\end{array}\right.
$$

in a complete Menger PN-space $(E, \mathcal{F}, \Delta)$ with a $t$-norm $\Delta$ satisfying $\Delta(t, t) \geq t$ for all $t \in[0,1]$. For any $\varepsilon>0$ and $\lambda>0$, we denote

$$
N\left(x_{0}, \varepsilon, \lambda\right)=\left\{x: F_{x-x_{0}}(\varepsilon)>1-\lambda\right\}, \quad x_{0} \in E .
$$

Let $f$ and $g$ be two continuous mappings from $R \times \overline{N\left(x_{0}, \varepsilon, \lambda\right)}$ into $E$ satisfying the following conditions:

(i) $F_{f(t, x)-f(t, y)}(s) \geq F_{x, y}(s / L)$ for all $s \geq 0$ and $x, y \in \overline{N\left(x_{0}, \varepsilon, \lambda\right)}$, where $L>0$ is a constant.

(ii) For each $t, g\left(t, \overline{N\left(x_{0}, \varepsilon, \lambda\right)}\right)$ is a relatively compact subset in $E$.

Theorem 5. Under the above conditions (i) and (ii), there exists a $\delta_{0}>0$ small enough such that the following differential equation:

$$
\left\{\begin{array}{l}
x^{\prime}(t)=f(t, x(t))+g(t, x(t)), \\
x(0)=x_{0}
\end{array}\right.
$$

has a solution in $C\left(\left[0, \delta_{0}\right], E\right)$.

Proof. We know that (3.12) is equivalent to the following integral equation:

$$
x(t)=x_{0}+\int_{0}^{t} f(s, x(s)) d s+\int_{0}^{t} g(s, x(s)) d s .
$$

Let $C\left(\left[0, \delta_{0}\right], E\right)$ be the same as above, and let

$$
W=\left\{x(\cdot) \in C\left(\left[0, \delta_{0}\right], E\right): x(0)=x_{0}, F_{x(s)-x_{0}}(\varepsilon)>1-\lambda, s \in\left[0, \delta_{0}\right]\right\},
$$

where $\delta_{0}>0$ is small enough such that $L \delta_{0}<1$ and

$$
F_{\int_{0}^{t}(f(s, x(s))+g(s, x(s))) d s}(\varepsilon)>1-\lambda, \quad t \in\left[0, \delta_{0}\right], x(\cdot) \in W .
$$


It is easy to see that $W \subset C\left(\left[0, \delta_{0}\right], E\right)$ is convex. Let

$$
T x(t)=x_{0}+\int_{0}^{t} f(s, x(s)) d s \quad \text { and } \quad A x(t)=\int_{0}^{t} g(s, x(s)) d s
$$

for all $x(\cdot) \in C\left(\left[0, \delta_{0}\right], E\right)$ and $x(s) \in \overline{N\left(x_{0}, \varepsilon, \lambda\right)}$ for all $s \in\left[0, \delta_{0}\right]$. We can prove that $T$ and $A$ are continuous and $T+A: W \rightarrow W$. Therefore, $T+A: \bar{W} \rightarrow \bar{W}$ and $A$ is compact.

Next we prove that $T: \bar{W} \rightarrow C\left(\left[0, \delta_{0}\right], E\right)$ is a generalized contractive mapping. In fact, for any $x(\cdot), y(\cdot) \in \bar{W}$, we have

$$
F_{T x(t)-T y(t)}(k)=F_{\int_{0}^{t}(f(s, x(s))-f(s, y(s))) d s}(k), \quad t \in\left[0, \delta_{0}\right] .
$$

Fro any given $t \in\left[0, \delta_{0}\right]$ and $k>0$, there exists a sequence $\left\{k_{t}^{(n)}\right\} \subset\left[0, \delta_{0}\right]$ which for all the continuous points of $F_{\int_{0}^{t} f(s, x(s))-f(s, y(s)) d s}(k)$ such that $k_{t}^{(n)} \rightarrow k-$ as $n \rightarrow+\infty$. Hence we have

$$
\begin{aligned}
& F_{T x(t)-T y(t)}(k) \geq F_{\int_{0}^{t}(f(s, x(s))-f(s, y(s))) d s}\left(k_{t}^{(n)}\right) \\
&=\max _{1 \leq i \leq N}\left(\Delta t_{i}\right) \rightarrow 0 \\
& \max _{\sum_{i=1}^{n}\left(f\left(\xi_{i}, x\left(\xi_{i}\right)\right)-f\left(\xi_{i}, y\left(\xi_{i}\right)\right)\right) \Delta t_{i}}\left(k_{t}^{(n)}\right),
\end{aligned}
$$

where

$$
0=\xi_{0}<\xi_{1}<\xi_{2}<\cdots<\xi_{N}=t, \quad \Delta t_{i}=\xi_{i}-\xi_{i-1}, \quad i=1,2, \ldots, N,
$$

and so it follows that

$$
\begin{aligned}
F_{T x(t)-T y(t)}(k) & \geq \lim _{\max _{1 \leq i \leq N}\left(\Delta t_{i}\right) \rightarrow 0} \min \left\{F_{f\left(\xi_{i}, x\left(\xi_{i}\right)\right)-f\left(\xi_{i}, y\left(\xi_{i}\right)\right)}\left(\frac{k_{t}^{(n)}}{t}\right)\right\} \\
& \geq \inf _{s \in\left[0, \delta_{0}\right]} F_{f(x, x(s))-f(x, y(s))}\left(\frac{k_{t}^{(n)}}{t}\right) \\
& \geq \inf _{s \in\left[0, \delta_{0}\right]} F_{x(s)-y(s)}\left(\frac{k_{t}^{(n)}}{L t}\right) \geq \inf _{s \in\left[0, \delta_{0}\right]} F_{x(s)-y(s)}\left(\frac{k_{t}^{(n)}}{L \delta_{0}}\right) .
\end{aligned}
$$

Letting $n \rightarrow \infty$ and taking the limit on the right side of (3.13), we have

$$
\begin{aligned}
F_{T x(t)-T y(t)}(k) & \geq \lim _{k_{t}^{(n)} \rightarrow k-}\left(\inf _{s \in\left[0, \delta_{0}\right]} F_{(s)-y(s)}\left(\frac{k_{t}^{(n)}}{L \delta_{0}}\right)\right) \\
& =\widetilde{F}_{x(\cdot)-y(\cdot)}\left(\frac{k}{L \delta_{0}}\right) .
\end{aligned}
$$

This implies that

$$
\inf _{t \in\left[0, \delta_{0}\right]} F_{T x(t)-T y(t)}(k) \geq \widetilde{F}_{x(\cdot)-y(\cdot)}\left(\frac{k}{L \delta_{0}}\right) .
$$

Thus we have

$$
\lim _{k \rightarrow \lambda-}\left(\inf _{t \in\left[0, \delta_{0}\right]} F_{T x(t)-T y(t)}(k)\right) \geq \lim _{k \rightarrow \lambda-} \widetilde{F}_{x(\cdot)-y(\cdot)}\left(\frac{k}{L \delta_{0}}\right),
$$

i.e.,

$$
\widetilde{F}_{T x(\cdot)-T y(\cdot)}(\lambda) \geq \widetilde{F}_{x(\cdot)-y(\cdot)}\left(\frac{\lambda}{L \delta_{0}}\right), \quad \lambda>0,
$$


which implies that $T: \bar{W} \rightarrow\left(C\left(\left[0, \delta_{0}\right], E\right), \widetilde{\mathcal{F}}, \Delta\right)$ is a generalized contractive mapping. Therefore, by Theorem 2 , the conclusion of Theorem 5 is proved. This completes the proof.

\section{ACKNowledgement}

These studies were supported in part by the Basic Science Research Institute Program, Ministry of Education, 1994, Project No. BSRI-94-1405 and NONDIRECTED RESEARCH FUND, Korea Research Foundation, 1994.

\section{REFERENCES}

1. S. S. Chang, On the theory of probabilistic metric spaces with applications, Z. Wahrsch. Gebiet 67 (1984), 85-94. MR 86a:54056

2. S. S. Chang, Y. Q. Chen, and J. L. Guo, Ekeland's variational principle and Caristi's fixed point theorem in probabilistic metric spaces, Acta Math. Appl. Sinica 7 (1991), 217-228. MR 93b: 49021

3. S. S. Chang, Y. J. Cho, and S. M. Kang, Probabilistic metric spaces and nonlinear operator theory, Sichuan Univ. Press, P. R. China, 1994.

4. B. Schweizer and A. Sklar, Statistical metric spaces, Pacific J. Math. 10 (1960), 313-334. MR 22:5955

5. B. Schweizer, A. Sklar, and E. Thorp, The metrization of statistical metric spaces, Pacific J. Math. 10 (1960), 673-675. MR 22:5956

(S. S. Chang and Y. Q. Chen) Department of Mathematics, Sichuan University, Chengdu, Sichuan 610064, People's Republic of China

(B. S. Lee) Department of Mathematics, Kyungsung University, Pusan 608-736, Korea E-mail address: bslee@ksmath.kyungsung.ac.kr

(Y. J. Cho and S. M. Kang) Department of Mathematics, Gyeongsang National University, Chinju 660-701, Korea

(J. S. Jung) Department of Mathematics, Dong-A University, Pusan 604-714, Korea

E-mail address: jungjs@seunghak.donga.ac.kr 